\title{
Sugar and reward system: Is high sugar intake generating akin effects like drugs of abuse?
}

\author{
Md. Sahab Uddin* \\ Department of Pharmacy, Southeast University, Dhaka, Bangladesh
}

Sugar is an imperative part of our diet. Only a tiny part of us pass a usual day deprived of adding sugar to typical meal. It is travail to exactly estimate sugar content in diet and food stuffs owing to its accessibility and affordability. Glucose, a genre of sugar, is the prime cradle of energy for every cell in the body [1]. Among the human body organs, the brain is the cardinal sugar energy-utilizing organ. Augmented sugar intake is considered one of the foremost factors for copious snags, including obesity and type II diabetes, but little is identified concerning the effects of enduring sugar intake on the brain.

Evidence proposes that high-sugar intake is accountable for intense neural vicissitudes in the brain areas intricate in reinforcement. The amygdala, particularly the basolateral amygdala (BLA) neurons is recognized to facilitate the reinforcing assets of drugs of abuse [2]. Ambroggi et al. reported that BLA input is requisite for dopamine to augment the firing of nucleus accumbens (NAc) [3]. The NAc is linked in the cognitive processing of reward, comprising particular penchant retorts to certain pleasant stimuli, motivational salience in addition to reinforcement $[4,5]$. The BLA gets dopaminergic input from the VTA and conveys glutamatergic projections to the medium spiny neurons (MSNs) in the NAc. MSNs are crucial assembly of the mesolimbic reward circuit [6].

Currently Shariff et al. examined the consequence of short- and long-term binge-like sucrose intake on the morphology of the BLA neurons by the help of an intermittent-access two-bottle choice paradigm [7]. The researchers used Golgi-Cox staining to impregnate principal neurons from the BLA of short- (i.e. 4 weeks) and long-term (i.e. 12 weeks) sucrose consuming adolescent rats and compared these to age-matched water controls. The denouements indicate possible maladaptive changes to the dendritic architecture of BLA principal neurons, particularly on apical dendrites following long-term sucrose consumption. Specifically, the consequences show reduced total dendritic arbor length of BLA principal neurons following short- and long-term sucrose intake. Furthermore, researchers found that longterm binge-like sucrose intake caused a significant reduction in the length and complexity of apical dendrites. Taken together, the results highlight the differences between short- and long-term binge-like sucrose intakes on BLA principal neuron morphology and are suggestive of a perturbation in the diverse synaptic inputs to these neurons.

Klenowski et al. stated that persistent intake of sucrose in a bingelike way, changes the morphology of medium spiny neurons of the NAc [8]. The conundrums presented ominously declined in the total dendritic length of NAc shell MSNs with respect to age-matched control rats owing to continual binge-like sucrose intake. They also reported that the reform of these neurons caused predominantly from abridged distal dendritic complexity. Contrariwise, in case of rats ingesting sucrose an increased in spine densities at the distal branch orders of NAc shell MSNs was observed. The concerns of this study augment excellence to the assumption that sugars (i.e. sucrose) have addictive possessions succeeding durable, binge-like intake (Figure 1).

Numerous studies have suggested that chronic acquaintance to drugs of abuse (i.e. alcohol, morphine, opium, cocaine, amphetamines) is accountable for cellular and morphological hallmark $[9,10]$ that are also similar to sugar. Not only sugar, but also high fat diet is also causative to generate addiction like state. In a study by Dingess et al. inspected exposure to high fat diet diminishes dendritic spine density in the medial prefrontal cortex [11]. Another study by Thompson et al. examined obesity-persuaded structural and neuronal plasticity in the lateral orbitofrontal cortex [12]. The outcomes of these studies suggested a substantial decrease in the density of spines and declined in inhibitory synaptic transmission in the prefrontal cortex which is profoundly linked with reward processing.

The comparison between sugar and drugs of abuse is a gullible alternative way. Undoubtedly, it is observed that natural rewards (i.e. sugar) and drugs of abuse (i.e. morphine, opium, cocaine, amphetamines) may share a few usual neuronal characteristics, but the real outcome of drugs of abuse is very precarious. This incongruity between sugar and drugs of abuse prompted structural alterations may

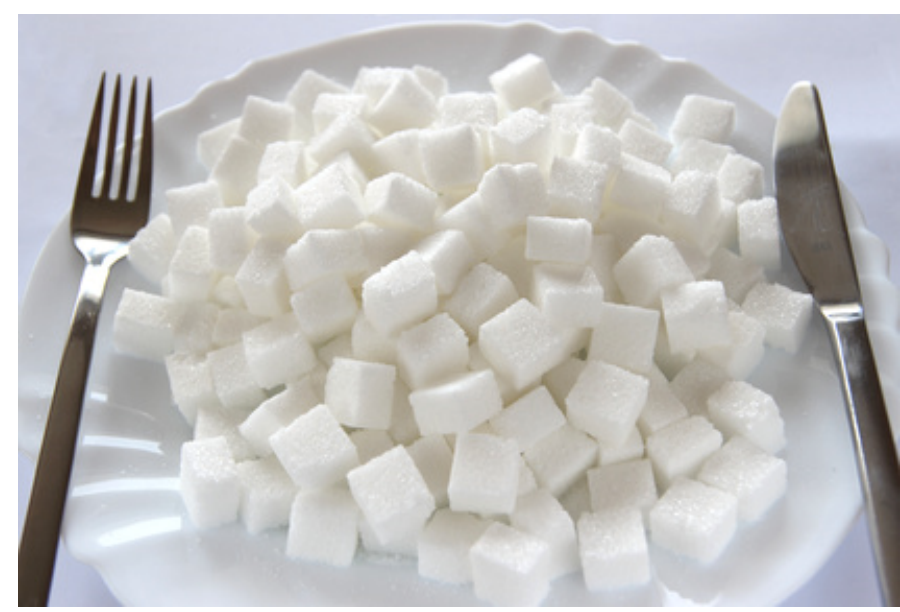

Figure 1. Sugar exerts a similar sort of addictive effect on society that drugs of abuse also reach.

Correspondence to: Md. Sahab Uddin, Department of Pharmacy, Southeast University, Dhaka, Bangladesh; Tel: +880 1710220110; E-mail: msu_ neuropharma@hotmail.com,msu-neuropharma@hotmail.com

Received: September 22, 2017; Accepted: October 17, 2017; Published: October 21, 2017 
specify that both rewards (i.e. sugar and drugs) may affect distinct landscapes of the neuronal homeostasis. However, further researches are indispensable to illustrate the exact mode of actions triggering sugar addiction facts.

\section{Acknowledgements}

The author wishes to thank the anonymous reviewer(s)/editor(s) of this article for their constructive reviews.

\section{Competing interests}

The author states no competing interests.

\section{References}

1. Satyanarayana U (2013) Biochemistry. 4th ed. India: Elsevier.

2. Wassum KM, Izquierdo A2 (2015) The basolateral amygdala in reward learning and addiction. Neurosci Biobehav Rev 57: 271-283. [Crossref]

3. Ambroggi F, Ishikawa A, Fields HL, Nicola SM (2008) Basolateral amygdala neurons facilitate reward-seeking behavior by exciting nucleus accumbens neurons. Neuron 59(4): 648-661. [Crossref]

4. Saddoris MP, Cacciapaglia F, Wightman RM, Carelli RM (2015) Differential dopamine release dynamics in the nucleus accumbens core and shell reveal complementary signals for error prediction and incentive motivation. $J$ Neurosci 35(33): 11572-11582. [Crossref]
5. Berridge KC, Kringelbach ML (2015) Pleasure systems in the brain. Neuron 86: 646664. [Crossref]

6. Adinoff B (2004) Neurobiologic processes in drug reward and addiction. Harv Rev Psychiatry 12: 305-320. [Crossref]

7. Shariff M, Klenowski P, Morgan M, Patkar O, Mu E, et al. (2017) Binge-like sucrose consumption reduces the dendritic length and complexity of principal neurons in the adolescent rat basolateral amygdala. Homberg J, ed. PLOS ONE 12(8): e0183063. [Crossref]

8. Klenowski PM, Shariff MR, Belmer A, Fogarty MJ, Mu EWH, et al. (2016) Prolonged Consumption of sucrose in a binge-like manner, alters the morphology of medium spiny neurons in the nucleus accumbens shell. Front Behav Neurosci 10(54): 1-10. [Crossref]

9. Gupta S, Kulhara P (2007) Cellular and molecular mechanisms of drug dependence: An overview and update. Indian J Psychiatry 49: 85-90. [Crossref]

10. Uddin MS, Sufian MA, Kabir MT, Hossain MF, Nasrullah M, et al. (2017) Amphetamines: Potent recreational drug of abuse. J Addict Res Ther 8(4): 1-12.

11. Dingess PM, Darling RA, Kurt Dolence E, Culver BW, Brown TE (2017) Exposure to a diet high in fat attenuates dendritic spine density in the medial prefrontal cortex. Brain Struct Funct 222(2): 1077-1085. [Crossref]

12. Thompson JL, Drysdale M, Baimel C, Kaur M, MacGowan T, et al. (2017) Obesity-induced structural and neuronal plasticity in the lateral orbitofrontal cortex. Neuropsychopharmacology 42(7): 1480-1490. [Crossref]

Copyright: $\odot 2017$ Uddin MS. This is an open-access article distributed under the terms of the Creative Commons Attribution License, which permits unrestricted use, distribution, and reproduction in any medium, provided the original author and source are credited. 